\title{
One Drug-Two Similar Reactions. Report of cases of Ciprofloxacin induced Stevens-Johnson Syndrome and Bullous fixed drug eruption
}

Sunder Nagakeerthana, Murugaiyan Rangaraj and Kaliaperumal Karthikeyan*

Department of Dermatology, Sri Manakula Vinayagar Medical College and Hospital, Kalitheerthalkuppam, Puducherry, INDIA.

\begin{abstract}
Ciprofloxacinis a broad spectrum quinolone antibiotic which is used to treat a wide variety of infections, especially those likely to be caused by Gramnegative bacteria. It acts by inhibiting DNA gyrase and topoisomerase IV enzymes which is necessary to separate bacterial DNA, thereby inhibiting cell division. It induces panoply of drug reactions ranging from an urticarial eruption to a lethal toxic epidermal necrolysis. We report two cases, Stevens-Johnson syndrome and Bullous fixed drug eruption induced by ciprofloxacin which can closely mimic each other. Though these drug reactions are caused by the same drug, the mechanism is different in SJS and FDE. As this drug can induce various adverse reactions, care should be taken while prescribing, and patient should be counseled about the possible side effects. Rapid recognition of patterns of adverse reactions are
\end{abstract}

important, besides the prompt withdrawal of the drug and specific treatment are the most essential action to decrease morbidity.

Key words: Ciprofloxacin, Stevens-Johnson syndrome, Fixed drug eruption, ALDEN, Naranjo algorithm.

Correspondence :

Dr. Kaliaperumal Karthikeyan,

Department of Dermatology, Sri Manakula Vinayagar Medical College and Hospital, Kalitheerthalkuppam, Puducherry, INDIA.

Phone no: +919443268208

E-mail: karthikderm@gmail.com

DOI: 10.5530/jyp.2016.2.22

\section{INTRODUCTION}

Ciprofloxacin belongs to a class of antibiotics called fluoroquinolones. It is a broad spectrum antibiotic used to treat a wide variety of infections, including lower and upper respiratory tract infections, urinary tract, skin and bone infections, and gastrointestinal tract infection especially those likely to be caused by Gram-negative bacteria. It acts by inhibiting DNA gyrase and topoisomerase IV enzymes which is necessary to separate bacterial DNA, thereby inhibiting cell division. ${ }^{1}$ Ciprofloxacin can produce a variety of adverse reactions. Cutaneous adverse reactions can range from mild urticarial reaction to more serious Toxic epidermal necrolysis. The type of reaction is influenced by genetic factors and the amount of drug taken. We report two cases, the first case was StevensJohnson syndrome and the second case was Bullous fixed drug eruption, both caused by ciprofloxacin which can closely mimic each other. In this report we discuss the differences between these types of cutaneous adverse reaction.

\section{CASE REPORT}

Case 1: A 43-year-old male presented to Dermatology Out-Patient Department (OPD) with complaints of painful lesions over bilateral hands, oral mucosa and genitalia for two days. Patient gave history of upper respiratory tract infection for which he was treated with Tab. Ciprofloxacin $500 \mathrm{mg}$ and Tab. levocetrizine $5 \mathrm{mg}$ twice daily. Two days later he developed fever, conjunctivitis, salivation and erythematous patches over oral cavity and genitalia which subsequently formed erosions with purulent discharge. On examination, multiple erosions with purulent discharge were seen over hard palate, upper and lower lips, angle of mouth (Figure 1). Erosions were also seen on penis and scrotum. Multiple, well defined erythematous patches were seen over dorsum of hands. Nikolsky's sign was negative. A clinical diagnosis of Stevens-Johnson syndrome was made. Patient was treated with Inj. Dexamethasone for 3 days and later tablet prednisolone. The patient gradually improved, and after 9 days of hospital care, he was discharged in good condition. The treatment with prednisolone was continued; the

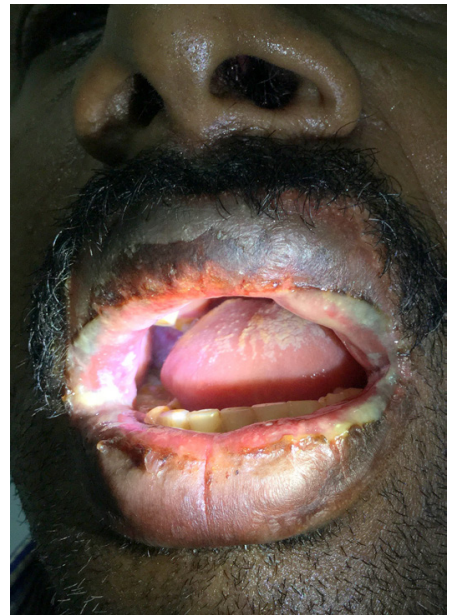

Figure 1: Erosions with purulent discharge seen over lips and oral

dosage of prednisolone was tapered and withdrawn after a period of 4 weeks. The patient is lesion free on follow up.

Case 2: A 27-year-old male presented to Dermatology OPD with complaints of multiple fluid filled lesions over both hands and erosions over face, lips and genitalia for two days. On detailed history patient revealed that he consumed over-counter medication of Tab. Ciprofloxacin $500 \mathrm{mg}$ twice daily for 3 days for upper respiratory tract infection. Following which he developed fluid filled lesions which spontaneously ruptured to form erosions. On examination, multiple vesicles and bullae interspersed with erosions and surrounding erythema seen over bilateral dorsum of hands (Figure 2). A well-defined erosion with surrounding erythema was seen over the prepuce. Multiple, well defined erythematous patches were seen over bilateral temple, upper and lower lips. Nikolsky's sign was negative. Clinical diagnosis of bullous fixed drug eruption was made; patient was treated as out-patient with tablet prednisolone and mometasone cream. 


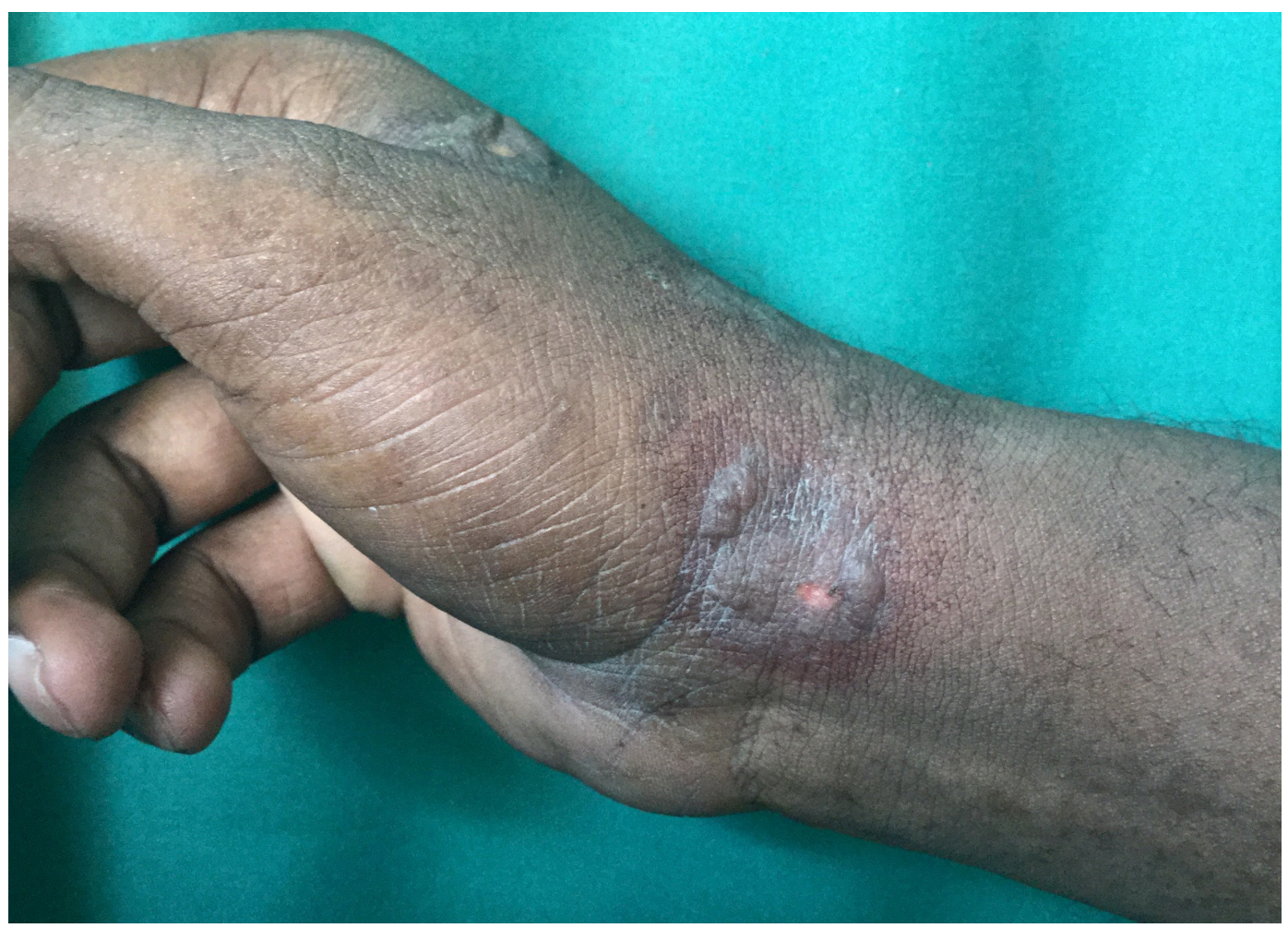

Figure 2: Bullous fixed drug eruption seen over the hand

Table 1: Cutaneous adverse drug reactions (CADRs) caused by Ciprofloxacin

\begin{tabular}{l}
\multicolumn{1}{c}{ Ciprofloxacin induced CADRs } \\
\hline 1. Bullous fixed drug eruption \\
2. Erythema multiforme \\
3. Vasculitis \\
4. Toxic epidermal necrolysis \\
5. Bullous Pemphigoid \\
6. Stevens-Johnson syndrome \\
7. DRESS \\
8. Urticaria \\
9. Sweets syndrome
\end{tabular}

Table 2: Differences between Bullous FDE and SJS

\begin{tabular}{|c|c|c|}
\hline Characteristics & Bullous Fixed drug eruption & Stevens-Johnson syndrome \\
\hline Incubation period & Within $10 \mathrm{~h}$ of drug intake & Few days to 1 month of drug intake \\
\hline Prodromal symptoms & Mild and shorter period & Severe flu like symptoms \\
\hline Lesions & Bullae and erythematous macules & Bullae, flat atypical targets or purpuric macules \\
\hline Distribution & Symmetrical & Predominantly central \\
\hline Skin & Predominantly involved & May be involved \\
\hline Mucosa & May be involved & Always involved \\
\hline On rechallenge & Recurs at the same site & No predilection for previously affected sites \\
\hline \multicolumn{3}{|l|}{ On histopathology } \\
\hline dermal melanophages & More & Lesser \\
\hline \multirow[t]{2}{*}{ Inflammatory infiltrate } & $\begin{array}{l}\text { Mixed inflammatory infiltrate containing } \\
\text { neutrophils and eosinophils }\end{array}$ & Lymphohistiocytic infiltrate \\
\hline & Seen around superficial and deep plexuses & Seen around superficial plexus \\
\hline Serum level of granulysin & Lower & Relatively higher \\
\hline Systemic complications & None & Present \\
\hline Sequelae & Post inflammatory hyperpigmentation & Ocular: Symphblepharon, trichiasis, xerosis \\
\hline
\end{tabular}


ALDEN and Naranjo algorithm indicated a probable relationship between the Ciprofloxacin therapy and the development of Stevens-Johnson syndrome in first patient and bullous fixed drug eruption in the next patient.

\section{DISCUSSION}

Ciprofloxacin is a well-known and widely used antibiotic for various infections. It induces panoply of drug reactions ranging from an urticarial eruption to a lethal toxic epidermal necrolysis. In a country where over counter prescription is rampant, the exact magnitude of the usage is very tough to estimate. It has been associated with the following types of drug reactions (Table 1). We encountered two types of drug reaction namely Stevens-Johnson syndrome (SJS) and Bullous Fixed Drug Eruption (FDE) which resembles each other closely. Though these drug reactions are caused by the same drug, the mechanism is different in SJS and FDE. SJS is an immune-complex mediated hypersensitivity disorder whereas FDE is due to delayed hypersensitivity reaction.

Stevens-Johnson syndrome-Toxic Epidermal Necrolysis (TEN) spectrum is one of the severe drug induced cutaneous reaction. SJS is the milder form; the incidence of SJS is estimated to be 1 to 6 cases per million people per year. It is characterized by erythema with blisters and erosions of the skin with the degree of epidermal detachment of less than $10 \%$ of body surface area, fever and malaise, and erosions of mucous membranes. ${ }^{2}$ Before the appearance of mucocutaneous erosions, the initial symptoms include fever, conjunctivitis, pharyngitis and pruritus. Mucous membranes in the oral cavity, eyes, genitalia, and anus are commonly affected a few days before skin lesions appear. The initial lesions are poorly defined macules with dark centers. The lesions usually reach their greatest area of coverage in a few days, but they can manifest over a period of a few hours after. ${ }^{3}$ Stevens-Johnson syndrome is an immunecomplex-mediated hypersensitivity disorder that may be caused by many drugs, viral infections, and malignancies. ${ }^{4}$ The main feature of SJS and TEN is epidermal cell apoptosis, which may be mediated through keratinocyte Fas-FasL interaction or through cytotoxic T-cell release of perforin and granzyme B. ${ }^{3}$

Fixed drug eruption (FDE) is a specific phenotype among adverse drug reactions affecting the skin. It is characterized by a limited number of round to oval erythematous patches that resolve in a few days, often leaving long-lasting pigmentation. Characteristic feature of FDE is recurrence on the same sites a few hours after rechallenge with the same medication. ${ }^{5}$ The exact mechanism for this FDE is still unclear but, it has been postulated that FDE is due to a delayed classical type IVc hypersensitivity reaction mediated by CD $8+\mathrm{T}$ cells. ${ }^{6,7}$ Several variants of FDE have been described, based on their clinical features and distribution of lesions including pigmented, generalized, linear, non-pigmented, bullous, eczematous, urticarial erythema dyschromicum perstans, vulvitis, psoriasiform or cellulitis. ${ }^{8}$ Bullous Fixed drug eruption may mimic SJS clinically. ${ }^{9}$ Though both our patients had clinical features which closely resembled each other, the morphology and mucous membrane involvement are useful in clinching diagnosis. SJS is different from Bullous FDE in many ways, it is important to distinguish SJS from Bullous FDE (Table 2). Rechallenging the patient to the suspected offending drug is the only known test to possibly discern the causative agent, but that is unethical and not advisable. Therefore, certain causality assessment scales regarding drug reaction have been described like the Naranjo ADR probability scale and ALDEN algorithm. ${ }^{10,11}$

Management of SJS depends upon the severity, in milder cases topical steroids and withdrawal of drug is enough but in severe cases fluid replacement, nutritional support, antibacterial therapy, ophthalmology consultation and early systemic corticosteroids are beneficial. A few studies have shown cyclosporine, plasmapheresis and IV immunoglobulin to be helpful. ${ }^{3}$ While FDE requires only topical steroids and symptomatic treatment.

\section{CONCLUSION}

Ciprofloxacin is a widely used quinolone for various infections. As this drug can induce various adverse reactions, care should be taken while prescribing, and patient should be counseled about the possible side effects. Rapid recognition of patterns of adverse reactions are important, besides the prompt withdrawal of the drug and specific treatment are the most essential action to decrease morbidity.

\section{CONFLICT OF INTEREST}

The authors declare no conflict of interest.

\section{ABBREVIATION USED}

SJS: Stevens-Johnson syndrome; TEN: Toxic epidermal necrolysis; FDE: Fixed drug eruption; OPD: Out-patient Department; ALDEN: Algorithm of drug causality assessment for SJS/TEN; CADRs: Cutaneous adverse drug reactions; FasL: Fas Ligand; ADR: Adverse drug reaction; IV: Intravenous; DNA: Deoxyribonucleic acid.

\section{REFERENCES}

1. Le Bel M. Ciprofloxacin: chemistry, mechanism of action, resistance, antimicrobial spectrum, pharmacokinetics, clinical trials, and adverse reactions. Pharmacotherapy. 1988;8(1):3-33

2. Roujeau JC, Kelly JP, Naldi L, Rzany B, Stern RS, Anderson T, et al. Medication use and the risk of Stevens-Johnson syndrome or toxic epidermal necrolysis. N Engl J Med. 1995;333(24):1600-8

3. Abe R. Toxic epidermal necrolysis and Stevens-Johnson syndrome: soluble Fas ligand involvement in the pathomechanisms of these diseases. J Dermatol Sci. 2008 Dec;52(3):151-9.

4. Shih-Chi S, Wen-Hung C. Update on pathobiology in Stevens-Johnson syndrome and toxic epidermal necrolysis. Dermatologica Sinica. 2013;31(4):175-80.

5. Kauppinen K, Stubb S. Fixed eruptions: causative drugs and challenge tests. Br J Dermatol. 1985;112(5):575-8.

6. Shiohara T, Mizukawa Y, TerakiY. Pathophysiology of fixed drug eruption: The role of skin-resident T cells. Curr Opin Allergy Clin Immunol. 2002;2(4):317-23.
7. Nitya S, Deepa K, Mangaiarkkarasi A, Karthikeyan K. Doxycycline induced generalized bullous fixed drug eruption-A case report. Journal of Young Pharmacists. 2013;5(4):195-6.

8. Ozkaya-Bayazit E, Bayazit H, Ozarmagan G. Drug related clinical pattern in fixed drug eruption. Eur J Dermatol. 2000;10(4):288-91.

9. Rai R, Jain R, Kaur I, Kumar B. Multifocal bullous fixed drug eruption mimicking Stevens-Johnson synrome. Indian J Dermatol Venereol Leprol. 2002;68(3):1756.

10. Naranjo CA, Busto U, Sellers EM, Sandor P, Ruiz I, Roberts EA, et al. A method for estimating the probability of adverse drug reactions. Clin Pharmacol Ther. 1981;30(2):239-45.

11. Sassolas B, Haddad C, Mockenhaupt M, Dunant A, Liss Y, Bork K, et al. ALDEN an algorithm for assessment of drug causality in Stevens-Johnson Syndrome and toxic epidermal necrolysis: comparison with case-control analysis. Clin Pharmacol Ther. 2010;88(1):60-8. 